\title{
Treating Neonatal Abstinence Syndrome from Clinical Perspectives
}

\author{
Fatemeh Nayeri, ${ }^{1}$ Bita Ebrahim, ${ }^{2}$ Mamak Shariat, ${ }^{2}$ Hosein Dalili, ${ }_{1}^{1}$ Majid Kalani, ${ }^{3}$ Niloofar Karbasian, ${ }^{2}$ \\ and Mahdi Sheikh, ${ }^{2}$ \\ ${ }^{1}$ Breastfeeding Research Center \\ ${ }^{2}$ Maternal, Fetal and Neonatal Research Center, Tehran University of Medical Sciences, Tehran, IR Iran \\ ${ }^{3}$ Pediatrics Department, Akbar-Abadi Hospital, Iran University of Medical Sciences, Tehran, IR Iran \\ "Corresponding author: Dr. Mahdi Sheikh, Maternal, Fetal and Neonatal Research Center, Vali-Asr Hospital, Imam Khomeini Hospital complexes, Keshavarz Blvd, Tehran, IR \\ Iran. Tel: +98-9128481663, E-mail: mahdisheikh@gmail.com
}

Received 2016 April 07; Revised 2017 May 27; Accepted 2017 June 17.

\begin{abstract}
Background: The Finnegan scoring system which is used to initiate and guide neonatal abstinence syndrome (NAS) therapy has several limitations that make it difficult to be used by many healthcare providers. Therefore in practice many experienced clinicians use their clinical judgements for initiating and continuation of NAS treatment.

Objectives: Assessing the degree of association between clinical judgments of experienced neonatologists and the Finnegan scoring system to initiate NAS treatment, and identifying the most prevalent items of the Finnegan scoring system which are independently associated with the need for NAS treatment.

Methods: This multi-centric prospective cohort study evaluated 60 admitted neonates who were born to illicit drugs dependent mothers and exhibited signs and symptoms of NAS. Neonates were evaluated based on the Finnegan scoring system once at birth and every four hours. Three consecutive Finnegan scores of $\geq 8$ or two consecutive Finnegan scores of $\geq 12$ were considered as the requirement for treatment with morphine. Five expert neonatologists with $>15$ years of experience, who were blinded to the Finnegan score results visited the patients and their clinical judgments for initiating the medical treatment for NAS were recorded. Results: Based on the Finnegan scoring system 26.7\%, and based on the clinical judgment 30\% of neonates required medical treatment. There was an excellent correlation between Finnegan scoring system and clinical judgment of experienced neonatologists $(\mathrm{r}=0.75, \mathrm{P}<0.001)$, which was highly sensitive (87.5\%) and specific $(90.9 \%)$ in detecting neonates with NAS who required medical treatment. Tremor $(\mathrm{P}<0.001)$, convulsion $(\mathrm{P}=0.001)$, projectile vomiting $(\mathrm{P}=0.001)$, increased muscle tonicity $(\mathrm{P}=0.02)$, tachypnea $(\mathrm{P}=0.04)$, and poor feeding $(\mathrm{P}=0.04)$ were the items of the Finnegan score that regardless of their severity were independently associated with the requirement for pharmacologic treatment.

Conclusions: In special circumstances when using Finnegan scoring system in regular basis is not applicable, initiating NAS treatment based on the clinical judgments of expert neonatologists can be acceptable. Tremor, convulsion, increased muscle tonicity, tachypnea, projectile vomiting, and poor feeding can be used for screening neonates after birth for early identification of opioidexposed infants who might require medical treatment.
\end{abstract}

Keywords: Addiction, Drug Dependency, Withdrawal

\section{Background}

Neonatal abstinence syndrome (NAS) consists of a spectrum of neurologic, gastrointestinal and metabolic signs and symptoms that typically occur during the first 48 - 72 postnatal hours in neonates who had prenatal opioid exposure (1-4). Studies in USA have revealed that from 2000 to 2009 , there was more than four-fold increase in the rate of prenatal maternal opioid use as well as three-fold increase in the incidence of NAS (5). With the increase of NAS incidence, its total hospital charges have also dramatically increased (5). Based on a recent report of national survey on drug use and health (NSDUH) in the United States 5.9\% of pregnant women are using illicit drugs during gestation (6). Among the neonates who were exposed to illicit drugs in utero, withdrawal signs and symptoms requiring medi- cal intervention develop in 27\% - 91\% (7).

Untreated NAS can cause significant morbidity and mortality (2). Defining the target population for treatment is of paramount importance, and deciding to start, adjust and terminate the treatment depends on the exact clinical evaluation of severity of signs caused by drug cessation (8). Different NAS scoring methods have been developed to assess the need for treatment in such neonates. The neonatal abstinence scoring system, often termed the "method of Finnegan" or the "Finnegan scoring system", which was proposed by Finnegan et al. (9), is widely used in neonatal units to initiate and guide NAS therapy, and has been shown to have a good sensitivity for identifying the 20 most common withdrawal signs in neonates (9). However, the Finnegan scoring system has several limitations that 
make it difficult to be used by many healthcare providers; it requires considerable training for reliable use, there are difficulties in judging some items accurately (e.g., the difference between "mild" and "marked" tremors) and different items weighting (10). Additionally the Finnegan scoring system is time consuming and is difficult to be carried out in short intervals, especially in the referral centers with limited numbers of healthcare providers. Finally, changes in neonatal care in which neonates now sleep on their back make the two items of the Finnegan scoring system that measure excoriation of the knees and nose less informative (11).

Due to the aforementioned reasons the scientists have tried to find a brief and easier method for determining the requirement for treatment in neonates with NAS, therefore many scoring systems were proposed; neonatal drug withdrawal scoring system (NDWSS) (also known as the Lipsitz Scale) (12), the Ostrea tool (13), neonatal narcotic withdrawal index (NNWI) (14), the neonatal withdrawal inventory (NWI) (15), the withdrawal assessment tool (WAT-1), opioid and benzodiazepine withdrawal scale (OBWS) (16), and the brief 3-item screening index (17), are among the proposed screening tools.

Well-trained and skillful nurses, with constant presence at the neonates' bedside for accurate observation, are required to use and work with the aforementioned methods to assess the requirement for NAS treatment, which is not practical due to the insufficient number of expert nurses and healthcare providers on each work shift in many neonatal wards, especially in the developing countries. Therefore, despite all the proposed measurements, many experienced clinicians, especially in the referral centers with limited human and facility resources, use their clinical judgements for initiating and continuation of NAS treatment.

We conducted this study to evaluate the degree of association between the clinical judgments of experienced neonatologists and the Finnegan scoring system to initiate NAS treatment, and to identify the most prevalent and discriminative items of the Finnegan scoring system which are independently associated with the need for treatment in NAS neonates.

\section{Methods}

\subsection{Study Population and Study Design}

This multi-centric prospective cohort study evaluated 60 neonates who were born to illicit drugs dependent mothers and exhibited signs and symptoms of NAS who were admitted to two referral centers from March 2014 to October 2015. Neonates were considered eligible if they were born within the study centers at $\geq 35$ weeks of gestation, had a history of exposure to addictive drugs in utero (determined by maternal history and confirmed by positive maternal urine toxicology screens during the last trimester of pregnancy), and demonstrated signs and symptoms of NAS. Exclusion criteria were having intra uterine growth retardation (IUGR), congenital anomalies, major concomitant medical illness requiring oxygen therapy, intravenous fluids or medications, neurologic abnormalities, and breastfed neonates.

After explaining the whole study, a written informed consent was obtained from the parents before enrolling their neonate in the study. The investigators were extensively trained prior to the initiation of the study in order to keep the consistency of calculating the Finnegan score within the study centers and minimize interpersonal bias. The trained investigators evaluated neonates and scored their clinical signs based on the Finnegan scoring system. The Finnegan scoring system was applied for each neonate once at birth and repeated on a regular basis, every four hours until no treatment was required. All the signs and scores in each checking time were recorded in separate sheets and kept until discharge. The treatment of neonates with NAS was based on the Finnegan scoring system. Three consecutive Finnegan scores of $\geq 8$ or two consecutive Finnegan scores of $\geq 12$, calculated every four hours were considered as the requirement for treatment. The neonates who required treatment were treated with oral morphine based on the standardized protocol demonstrated in Table 1 (18). Treatment with oral morphine continued until Finnegan score remained below 8 for 72 hours then the drug was tapered gradually by $10 \%$ of maximal dose daily until it was entirely discontinued at the cessation dose of $0.1 \mathrm{mg} / \mathrm{kg} / 24$ hours of morphine (18). In addition to the investigators, five expert neonatologists with $>15$ years of experience in clinical practice as neonatologist in referral centers, visited the patients on a daily basis and their opinions for initiation of the medical treatment for NAS based on their clinical judgement were recorded. The correlation between the Finnegan scoring system and the clinical judgement of the experienced neonatologists regarding the requirement for NAS treatment were investigated. This study was approved by the ethics committee of our institute.

\subsection{Statistical Methods - Statistical Analysis}

Sample size was calculated for a power of $80 \%, \alpha=0.05$, $\beta=20 \%$. All the statistical analyses were performed using SPSS statistical software (version 18.0.0: PASW, Chicago, IL). The Pearson correlation coefficient, Chi-square analysis, Fisher's exact test, independent-samples t-test, logistic 
Table 1. The Protocol of Treatment with Oral Morphine Sulfate

\begin{tabular}{|c|c|c|}
\hline Finnegan Score & $0.4 \mathrm{mg} / \mathrm{mL}$ Diluted Morphine Sulfate, $\mathrm{mL} / \mathrm{kg} / 24 \mathrm{~h}$ & Morphine Sulfate, $\mathrm{mg} / \mathrm{kg} / 24 \mathrm{~h}$ \\
\hline $8-10$ & 0.6 & 0.24 \\
\hline $11-13$ & 1.2 & 0.48 \\
\hline 14 - 16 & 1.6 & 0.64 \\
\hline 17 and above & 2 & 0.8 \\
\hline \multicolumn{3}{|c|}{ Continued until Finnegan score remained below 8 for $72 \mathrm{~h}$ then } \\
\hline \multicolumn{3}{|c|}{ The drug was weaned gradually by $10 \%$ of maximal dose every day until it was entirely discontinued } \\
\hline \multicolumn{3}{|c|}{ Cessation dose: less than $0.1 \mathrm{mg} / \mathrm{kg} / 24 \mathrm{~h}$} \\
\hline
\end{tabular}

regression model, sensitivity, specificity, positive and negative predictive values and likelihood ratios were used for analyzing the data. The level of statistical significance was set at $P$ value $<0.05$.

\section{Results}

Seventy neonates were eligible to participate in the study, 10 were excluded of whom 5 were breastfed; 3 had IUGR; and 2 required intravenous fluids and medication due to sepsis. 60 neonates who were born to illicit drugs dependent mothers and exhibited signs and symptoms of NAS remained in the study. Upon enrollment the mean \pm standard deviation (SD) for maternal age was $27.8 \pm 3.1$ years; gestational age $38.5 \pm 2.3$ weeks; birth weight 2759 \pm 588 grams; 1 -minute Apgar score $8.4 \pm 1.3$; 5-minute Apgar score $9 \pm 1.4$. Seventeen (28.3\%) mothers were smokers, 31 (51.7\%) neonates were male, 30 (50\%) had been delivered through cesarean section. Opium (36.7\%) and cocaine $(36.7 \%)$ were the most common illicit drugs abused by mothers, followed by methadone (11.7\%), and polysubstance (10\%). Heroin was the least (5\%) common abused drug.

Based on the Finnegan scoring system 16 (26.7\%) neonates required medical treatment while according to the clinical judgment of the neonatologists 18 (30\%) neonates required treatment for NAS. There was an excellent significant correlation between Finnegan scoring system and clinical judgment of neonatologists regarding the initiation of treatment for NAS $(r=0.75, \mathrm{P}<0.001)$. The clinical judgment of experienced neonatologists for the initiation of NAS treatment was highly sensitive (87.5\%, 95\% CI $=61.6$ - 98.4) and specific $(90.9 \%, 95 \% \mathrm{CI}=78.3-97.4)$, with a positive predictive value of $77.8 \%$ ( $95 \% \mathrm{CI}=52.3-93.5)$, a negative predictive value of $95.2 \%(95 \% \mathrm{CI}=83.8-99.4)$, a positive likelihood ration of $9.62(95 \% \mathrm{CI}=3.7-24.9)$, and negative likelihood ratio of $0.14(95 \% \mathrm{CI}=0.04-0.5)$ for detecting neonates with NAS who require medical treatment based on the Finnegan scoring system.

We used logistic regression model to assess the most important signs and symptoms of NAS that are independently associated with the requirement for treatment based on the Finnegan scoring system; tremor $(\mathrm{B}=5.02$, $\mathrm{OR}=25.5,95 \% \mathrm{CI}=19.44-32.16, \mathrm{P}<0.001)$, convulsion $(\mathrm{B}=$ 3.06, $\mathrm{OR}=21.45,95 \% \mathrm{CI}=3.26-141.55, \mathrm{P}=0.001)$, projectile vomiting $(\mathrm{B}=1.58, \mathrm{OR}=4.86,95 \% \mathrm{CI}=1.86-12.65, \mathrm{P}=0.001)$, increased muscle tonicity $(\mathrm{B}=1.29, \mathrm{OR}=3.63,95 \% \mathrm{CI}=1.15$ $11.43, \mathrm{P}=0.02)$, tachypnea $(\mathrm{B}=2.38, \mathrm{OR}=10.84,95 \% \mathrm{CI}=1.07$ 109.29, $\mathrm{P}=0.04)$, and poor feeding $(\mathrm{B}=1.37, \mathrm{OR}=3.93,95 \% \mathrm{CI}$ $=1.06-16.02, \mathrm{P}=0.04)$.

\section{Discussion}

In this multi-centric prospective cohort study we evaluated 60 neonates with NAS, born to mothers with illicit drug abuse. Based on the Finnegan scoring system 16 (26.7\%) neonates required medical treatment while based on the clinical judgment of the neonatologists 18 (30\%) neonates required treatment for NAS. This was in accordance with the study of Strauss et al that evaluated 72 pregnant illicit drugs dependent mothers of whom $30 \%$ of their neonates required pharmacologic treatment with NAS (19). However in some other studies up to $90 \%$ of the neonates were reported to require pharmacologic treatment for NAS $(7,20)$. The treatment rate varies depending on the type of drugs abused by mothers; the requirement for pharmacologic treatment is usually higher among poly substance and opioid exposed neonates while it is lower in stimulants only exposed neonates whose the majority do not require pharmacologic treatment $(7,18)$.

In the current study tremor, convulsion, increased muscle tonicity, tachypnea, projectile vomiting, and poor feeding were the most important signs and symptoms of NAS, presence of which, regardless of their intensity and scale, was independently associated with the requirement for NAS treatment. Similarly in the study of Lipsitz, mus- 
cle tremor, increased muscle tonicity, tachypnea, irritability and intense crying were identified as the most important and prevalent signs (2). In Jones et al. study, a 3-sign index, consisting of hyperactive Moro reflex, mild tremors when undisturbed, and increased muscle tone, showed excellent discrimination, and the authors suggested using this 3-sign index for screening the neonates for NAS (17). Based on the studies that found Finnegan scoring system to be too complex by many nurseries for routine use (10), along with the aforementioned studies (12,17), we suggest that using the identified signs and symptoms for screening neonates after birth may provide a cost-effective mechanism for the identification of opioid-exposed infants in crowded centers where insufficient number of expert nurses and healthcare providers are present.

In this study for the first time we evaluated the predictive values of the expert neonatologists for initiating NAS treatment, and assessed the association between expert opinion and the Finnegan scoring system for initiating NAS treatment. This study showed that there is excellent correlation between experts' opinion and the Finnegan score for initiating the NAS treatment, in addition, the clinical judgment of experienced neonatologists was highly sensitive and specific with very good predictive values in detecting the neonates with NAS who required treatment based on the Finnegan scoring system. It should be noted that this study never intended to support the replacement of Finnegan scoring system by clinical judgments of experienced neonatologists. Due to the difficulties and time consuming nature of the Finnegan scoring system, many experienced clinicians, especially in the referral centers with limited human and facility resources, use their clinical judgement for initiating and continuation of NAS treatment. This study aimed to assess the accuracy of clinical opinions of the experienced neonatologists, and to evaluate whether ethically this method is acceptable. The results of this study documented that in special circumstances when using Finnegan scoring system in regular basis is not applicable, initiating NAS treatment based on the clinical judgment of expert neonatologists is acceptable and ethical because of its high sensitivity and specificity and excellent correlation with the Finnegan scoring system.

\section{References}

1. Jones HE, Finnegan LP, Kaltenbach K. Methadone and buprenorphine for the management of opioid dependence in pregnancy. Drugs. 2012;72(6):747-57. doi: 10.2165/11632820-000000000-00000. [PubMed: 22512363].

2. Gordon AL, Lopatko OV, Haslam RR, Stacey H, Pearson V, Woods $\mathrm{A}$, et al. Ineffective morphine treatment regimen for the control of Neonatal Abstinence Syndrome in buprenorphine- and methadone-exposed infants. J Dev Orig Health Dis. 2012;3(4):262-70. doi: 10.1017/S2040174412000190. [PubMed: 25102147].
3. Jones HE, Kaltenbach K, Heil SH, Stine SM, Coyle MG, Arria AM, et al. Neonatal abstinence syndrome after methadone or buprenorphine exposure. N Engl J Med. 2010;363(24):2320-31. doi: 10.1056/NEJMoa1005359. [PubMed: 21142534].

4. Rayburn WF, Bogenschutz MP. Pharmacotherapy for pregnant women with addictions. Am J Obstet Gynecol. 2004;191(6):1885-97. doi: 10.1016/j.ajog.2004.06.082. [PubMed: 15592269].

5. PatrickSW, Schumacher RE, Benneyworth BD, Krans EE, McAllister JM, Davis MM. Neonatal abstinence syndrome and associated health care expenditures: United States, 2000-2009. JAMA. 2012;307(18):1934-40. doi: 10.1001/jama.2012.3951. [PubMed: 22546608].

6. Substance Abuse and Mental Health Services Administration . Results from the 2005 national survey on drug use and health: national findings. Rockville: Substance Abuse and Mental Health Services Administration; 2013.

7. Kocherlakota P. Neonatal abstinence syndrome. Pediatrics. 2014;134(2):e547-61. doi: 10.1542/peds.2013-3524. [PubMed: 25070299].

8. Hudak ML, Tan RC, Committee On D, Committee On F, American Academy of P. Neonatal drug withdrawal. Pediatrics. 2012;129(2):e540-60. doi: 10.1542/peds.2011-3212. [PubMed: 22291123].

9. Finnegan LP, Connaughton JJ, Kron RE, Emich JP. Neonatal abstinence syndrome: assessment and management. Addict Dis. 1975;2(1-2):14158. [PubMed: 1163358].

10. McQueen K, Murphy-Oikonen J. Neonatal Abstinence Syndrome. $N$ Engl J Med. 2016;375(25):2468-79. doi: 10.1056/NEJMra1600879. [PubMed: 28002715].

11. Zimmermann-Baer U, Notzli U, Rentsch K, Bucher HU. Finnegan neonatal abstinence scoring system: normal values for first 3 days and weeks 5-6 in non-addicted infants. Addiction. 2010;105(3):524-8. doi: 10.1111/j.1360-0443.2009.02802.x. [PubMed: 20402996].

12. Lipsitz PJ. A proposed narcotic withdrawal score for use with newborn infants. A pragmatic evaluation of its efficacy. Clin Pediatr (Phila). 1975;14(6):592-4. doi: 10.1177/000992287501400613. [PubMed: 1126108].

13. Ostrea EM, Chavez CJ, Strauss ME. A study of factors that influence the severity of neonatal narcotic withdrawal.JPediatr. 1976;88(4 Pt1):6425. [PubMed: 1255327].

14. Green M, Suffet F. The Neonatal Narcotic Withdrawal Index: a device for the improvement of care in the abstinence syndrome. Am J Drug Alcohol Abuse. 1981;8(2):203-13. [PubMed: 7331976].

15. Zahorodny W, Rom C, Whitney W, Giddens S, Samuel M, Maichuk G, et al. The neonatal withdrawal inventory: a simplified score of newborn withdrawal. J Dev Behav Pediatr. 1998;19(2):89-93. [PubMed: 9584937].

16. Franck LS, Harris SK, Soetenga DJ, Amling JK, Curley MA. The Withdrawal Assessment Tool-1 (WAT-1): an assessment instrument for monitoring opioid and benzodiazepine withdrawal symptoms in pediatric patients. Pediatr Crit Care Med. 2008;9(6):573-80. doi: 10.1097/PCC.0b013e31818c8328. [PubMed: 18838937].

17. Jones HE, Harrow C, O'Grady KE, Crocetti M, Jansson LM, Kaltenbach K. Neonatal abstinence scores in opioid-exposed and nonexposed neonates: a blinded comparison. J Opioid Manag. 2010;6(6):409-13. [PubMed: 21269001].

18. Nayeri F, Sheikh M, Kalani M, Niknafs P, Shariat M, Dalili H, et al. Phenobarbital versus morphine in the management of neonatal abstinence syndrome, a randomized control trial. BMC Pediatr. 2015;15:57. doi: 10.1186/s12887-015-0377-9. [PubMed: 25976238].

19. Strauss ME, Andresko M, Stryker JC, Wardell JN, Dunkel LD. Methadone maintenance during pregnancy: pregnancy, birth, and neonate characteristics. Am J Obstet Gynecol. 1974;120(7):895-900. [PubMed: 4429107].

20. Coyle MG, Ferguson A, Lagasse L, Oh W, Lester B. Diluted tincture of opium (DTO) and phenobarbital versus DTO alone for neonatal opiate withdrawal in term infants. J Pediatr. 2002;140(5):561-4. doi: 10.1067/mpd.2002.123099. [PubMed: 12032522]. 